\title{
Fabrication of a centimeter-long cavity on a nanofiber for cavity QED
}

\author{
Jameesh Keloth, K. P. Nayak, and K. Hakuta
}

\begin{abstract}
We report the fabrication of a $1.2 \mathrm{~cm}$ long cavity directly on a nanofiber using femtosecond laser ablation. The cavity modes with finesse value in the range 200-400 can still maintain the transmission between 40-60\%, which can enable "strong-coupling" regime of cavity QED for a single atom trapped $200 \mathrm{~nm}$ away from the fiber surface. For such cavity modes, we estimate the one-pass intra-cavity transmission to be $99.53 \%$. Other cavity modes, which can enable high cooperativity in the range 3-10, show transmission over $60-85 \%$ and are suitable for fiber-based single photon sources and quantum nonlinear optics in the "Purcell" regime.
\end{abstract}

Efficient quantum state transfer between single photons and single atom is a key challenge towards realization of quantum networks [1]. Interaction of a single atom with strongly confined photons in an optical cavity leading to cavity QED effects, is a promising approach to realize a quantum interface [1-3]. A crucial requirement to achieve strong interaction between single photon and single atom, is that the single atom cooperativity parameter $C=\left(2 g_{0}\right)^{2} /\left(\kappa \gamma_{0}\right)>>1$, where $2 g_{0}$ is the single photon Rabi frequency, $\kappa$ is the cavity decay rate (linewidth) and $\gamma_{0}$ is the atomic spontaneous emission rate in vacuum. Even with $C>>1$, there are two regimes with different dynamics, a) "Purcell" regime, when $\kappa>2 g_{0}, \gamma_{0}$ and b) "strong-coupling" regime, when $2 g_{0}>\kappa, \gamma_{0}$. The "strong-coupling" regime has been investigated using free-space Fabry-Perot (FP) cavities, where the coherent quantum phenomena like single-atom lasing and vacuum Rabi oscillations have been demonstrated [ $[3-5]$. However, it requires extremely high finesse of 0.3 to 0.5 million, which is technically challenging. Although high quality mirrors with transmission and scattering loss less than $2 \mathrm{ppm}$ has been reported, but the overall cavity transmission may drop to $10-20 \%$ [3, 6]. Following the development of free-space FP cavities, various designs of nanophotonic cavities have also been developed and investigated. In particular, the designs have focused on the "Purcell" regime, for applications like single photon generation, single photon switching and quantum nonlinear optics, where high transmission is essential [3, 7].

In the vision of a quantum network, efficient integration of the quantum interface to the existing fiber network is also an essential requirement. In this context, optical nanofiber based cavities offer a flexible alternative platform [8]. The nanofiber is the subwavelength diameter waist of a tapered single mode optical fiber. Using adiabatic tapering condition [9], efficient mode coupling to nanofiber region can be realized [10], enabling efficient integration to fiber networks. Strong transverse confinement of guided fields down to wavelength scale can be realized in the nanofiber. Moreover a major part of the guided field propagates outside the fiber enabling interaction with the surrounding medium. In order to get insight about the interaction dynamics in a nanofiber based cavity we follow the formalism developed in ref. [8]. Based on the formalism, $2 g_{0}=2 \sqrt{\eta \gamma c / L}$ and $\kappa=\pi c /(F L)$, re- spectively, where $\eta$ is the channeling efficiency of spontaneous emission of atom into the nanofiber guided modes without a cavity, $\gamma$ is the atomic spontaneous emission rate near the nanofiber, $c$ is the speed of light in vacuum, $L$ is the optical length of the cavity and $F$ is the finesse of the cavity mode. From this one can get, $C=\left(2 g_{0}\right)^{2} /\left(\kappa \gamma_{0}\right) \simeq 4 \eta F / \pi$. One should notice that the $C$ is independent of $L$ and mainly depends on $F$ and the transverse confinement of the optical mode through $\eta$. The effective mode waist of the nanofiber or other nanophotonic structures can be less than $1 \mu \mathrm{m}$ which is one order smaller compared to the typical mode waist of $10-30 \mu \mathrm{m}$ for free space FP cavities. As a result high cooperativity can be achieved even for moderate finesse of 50 - 100. Furthermore in case of nanofiber cavities one can independently control the cavity length to reach the "strong-coupling" regime since the $\kappa$ value reduces faster than the $2 g_{0}$ value as the cavity length increases.

Fabrication of cavity structures on the nanofiber using the focused ion beam milling has been demonstrated 11]. Also fabrication of the photonic crystal nanofiber (PhCN) cavity using the femtosecond laser ablation has already been demonstrated [12, 13]. A composite photonic crystal nanofiber cavity is also demonstrated by mounting a nanofiber on a nanofabricated grating structure and using single quantum dot on such a cavity, Purcell enhancement factor of 7 has been demonstrated [14, 15]. In the above cases, the cavity is formed directly on the nanofiber and designed for operation in "Purcell" regime with high transmission of up to $80 \%$. The cavity lengths ranged from $30 \mu \mathrm{m}$ to few $\mathrm{mm}$, depending on the cavity design and mainly limited by the nanofiber length of few $\mathrm{mm}$. On the other hand, extremely long nanofiber cavities with cavity length of 10-33 cm are also realized by splicing two conventional single mode fiber Bragg gratings to the tapered fiber [16, 17]. In this type of cavities, the presence of the tapered section within the cavity may induce intra-cavity loss and limit the achievable finesse and on-resonance transmission. The one-pass intra-cavity transmission reported in ref. [16] and [17] are $98.3 \%$ and $94 \%$, respectively. Hence for a finesse in the range 50 - 100 the total cavity transmission may reduce to $10 \%$ in ref. [16] and below $5 \%$ in ref. [17]. Nevertheless, using such extremely long nanofiber cavity, strongcoupling between single trapped Cs-atoms and the cavity guided photons have been demonstrated [17]. 


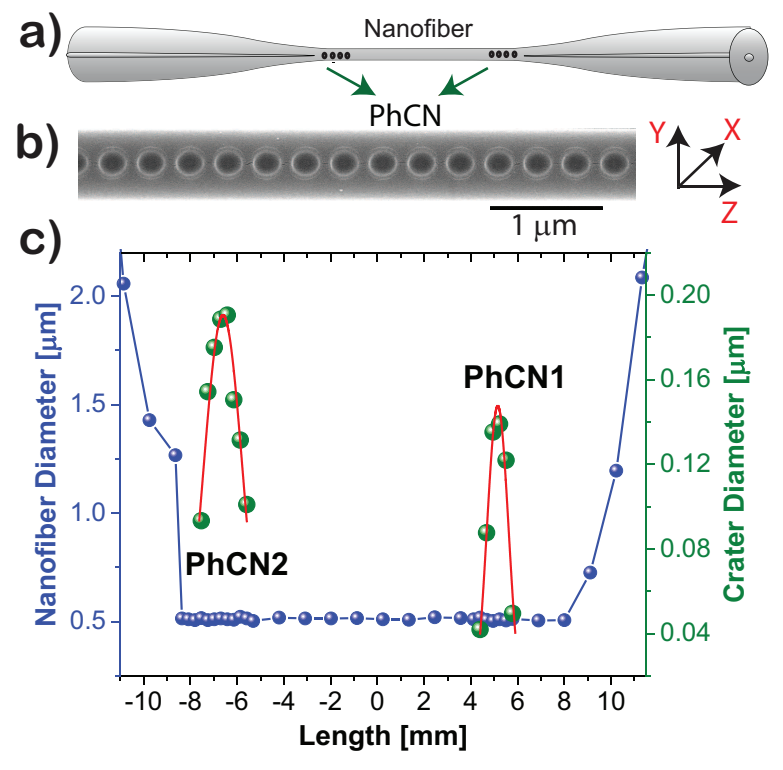

FIG. 1. (a) Schematic diagram of the long nanofiber cavity. (b) SEM image of a typical fabricated PhCN sample. (c) Diameter profiles of the nanofiber (blue circles) and the nanocraters (green circles) fabricated on it. The red curves are the Gaussian fit to the nano-crater profiles. The blue line is guide to the eyes.

In this letter, we report the fabrication of a centimeterlong cavity directly on the nanofiber, which can operate both in "Purcell" and "strong-coupling" regimes of cavity QED. We demonstrate the fabrication of a $1.7 \mathrm{~cm}$ long nanofiber with highly uniform diameter of $500 \pm 2$ $\mathrm{nm}$ over the entire length and maintaining high transmission of $>99 \%$, which is a crucial requirement for this approach. Furthermore, we fabricate two photonic crystal structures separated by $1.2 \mathrm{~cm}$ on such a nanofiber using femtosecond laser ablation, thus forming a long nanofiber cavity. The cavity modes with finesse value in the range 200-400 can still maintain the transmission between 40-60\%, enabling "strong-coupling" regime for a single atom trapped $200 \mathrm{~nm}$ away from the fiber surface [8]. For such cavity modes, we estimate the onepass intra-cavity transmission to be $99.53 \%$. Other cavity modes, which can enable high cooperativity in the range $3-10$, show transmission over $60-85 \%$ and are suitable for quantum nonlinear optics in the "Purcell" regime. Moreover, placing solid-state quantum emitters directly on the nanofiber surface such cooperativity will be further enhanced by a factor of 5 , which will be promising for fiber-based single photon sources.

Figure 1(a) shows the schematic diagram of the nanofiber cavity. The nanofiber is fabricated by tapering a standard single mode optical fiber using heat-and-pull technique [18]. The nanofiber is located at the waist of the tapered optical fiber. In order to extend the nanofiber length from few millimeters to few centimeters, we imple-

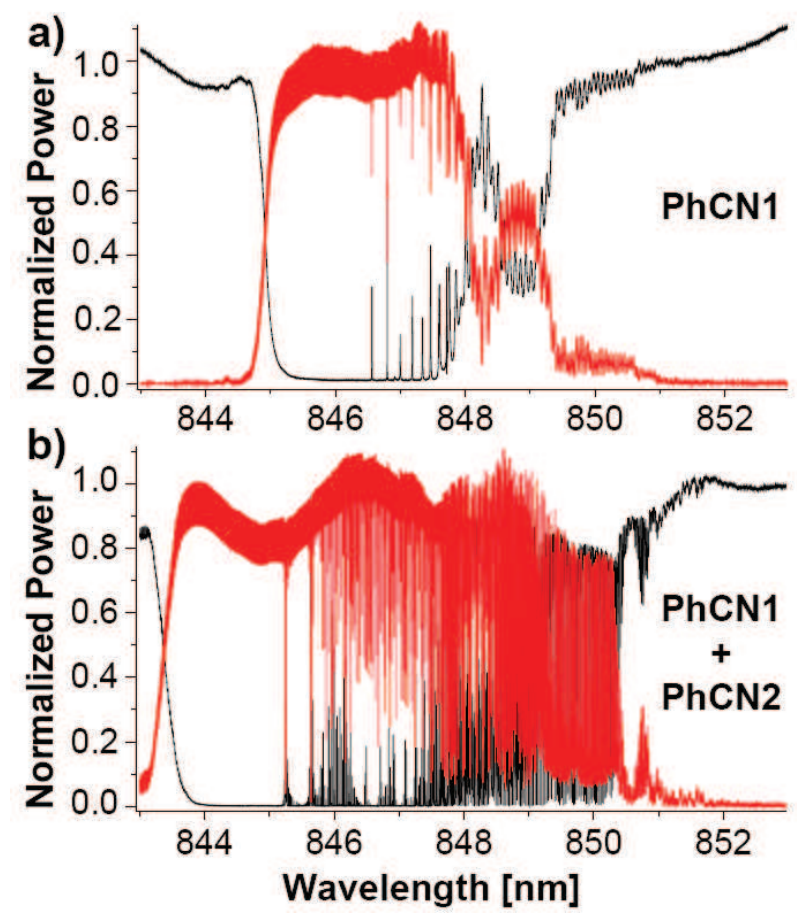

FIG. 2. Transmission (black lines) and reflection (red lines) spectra of the nanofiber after the fabrication of the (a) first and (b) second PhCNs. The spectra are measured for one of the principal polarizations (X-pol).

ment the linearly increasing hot-zone technique [18]. The profile of the tapered fiber and the pulling parameters are designed based on the adiabatic tapering guidelines detailed in ref. [9, 18]. By optimizing the parameters we have realized $>99 \%$ transmission for tapered fibers with a total length of 7 to $8 \mathrm{~cm}$, with a nanofiber waist diameter of $500 \mathrm{~nm}$ and waist length of 1.5 to $2.5 \mathrm{~cm}$. The uniformity of the diameter over the entire length of the nanofiber is $\pm 2 \mathrm{~nm}$ which is measured using a nondestructive and in situ method detailed in ref. [19]. The photonic crystal structures are fabricated at the two ends of the nanofiber using the femtosecond laser ablation [12, 13], thus forming a long nanofiber cavity. The scanning electron microscope image of a typical part of the $\mathrm{PhCN}$ is shown in Fig. 1(b). One can see that periodic nano-crater structures with a period of $350 \mathrm{~nm}$ are fabricated on the nanofiber [12, 13]. Figure 1(c) shows the diameter profile of a long nanofiber cavity whose optical properties are discussed in the following paragraphs. It shows the diameter profile of the nanofiber (blue circles) and that of the nano-craters (green circles) fabricated on it. The nanofiber diameter is $500 \mathrm{~nm}$ and it is uniform over a length of $1.7 \mathrm{~cm}$. The two $\mathrm{PhC}$ structures (indicated as PhCN1 and PhCN2) are fabricated on the nanofiber with a separation of $1.2 \mathrm{~cm}$. The PhCN1 is fabricated first and the PhCN2 is fabricated later. Each $\mathrm{PhCN}$ structure consists of thousands of periodic nanocraters [12, 13]. The diameter profiles of the nano-craters 
show a peak-like structure. The diameters at the peak of the profiles for the PhCN1 and PhCN2 are $140 \mathrm{~nm}$ and $190 \mathrm{~nm}$, respectively. The red curves show the Gaussian fits to the diameter profiles yielding the $1 / \mathrm{e}^{2}$-widths of $0.9 \mathrm{~mm}$ and $1.7 \mathrm{~mm}$ for the PhCN1 and PhCN2, respectively. This suggests that the second fabrication was stronger than the first one.

The transmission and reflection spectra of the long nanofiber cavity are measured using a tunable, narrow linewidth diode laser (Newport TLB6700). The laser is coupled to the tapered fiber through a 99:1 fiber-inline beam splitter. The input light is launched through the $1 \%$ port and the transmission is measured after the tapered fiber, whereas the reflection is measured through the $99 \%$ port in the reverse direction. The spectra are recorded by monitoring the power in the transmission and reflection ports using photodiodes while the laser frequency is being scanned. The polarization control is achieved using a fiber-inline polarizer before the tapered fiber. Figure 2(a) shows the transmission (black curve) and reflection (red curve) spectra after the fabrication of the $\mathrm{PhCN} 1$ for the polarization perpendicular to the nano-crater faces (X-pol). One can see that the stopband extends over $845 \mathrm{~nm}$ to $848 \mathrm{~nm}$, where the light is strongly reflected back. Also one can notice that sharp cavity modes appear in the red-side of the stopband. The mode spacing is $95.5 \mathrm{GHz}$ corresponding to a cavity length of $1.3 \mathrm{~mm}$. As discussed in the ref. [12, 13], those cavity modes are due to the apodized index variation along the PhCN1. Figure 2(b) shows the transmission (black curve) and reflection (red curve) spectra after the fabrication of both the PhCN1 and PhCN2. One can see that the width of the stopband increased and extends over $844 \mathrm{~nm}$ to $850 \mathrm{~nm}$. Moreover many closely spaced cavity modes appeared. This suggests that the second fabrication was stronger and confirms proper overlap between the stopbands of PhCN1 and PhCN2 to form a long nanofiber cavity.

In order to properly resolve the cavity modes and precise normalization of the on-resonance transmission and reflection values, we use a $\mathrm{CW}$ Ti-sapphire laser source (MBR-110, Coherent Inc.). In this measurement the laser frequency is locked to a reference cavity and the linewidth is $100 \mathrm{kHz}$. The spectra are recorded by monitoring the power in the transmission and reflection ports while stretching the tapered fiber. The details of such a technique was reported in ref.[13]. Although we chose this method to be more reliable, we must mention that the spectra measured using laser frequency scanning technique yielded similar results with only marginal differences. A typical part of the transmission (black curve) and reflection (red curve) spectra for X-pol is shown in Fig. 3(a). One can see periodically spaced sharp cavity modes. The mode spacing $\left(\Delta \nu_{F S R}\right)$ is 10.36 GHz. From the $\Delta \nu_{F S R}$ value we estimate a cavity length $\left(l=L / n_{\text {eff }}=c /\left(2 n_{\text {eff }} \Delta \nu_{F S R}\right)\right)$ of $1.2 \mathrm{~cm}$, where $n_{\text {eff }}$ $(\simeq 1.2)$ is the effective index of the nanofiber guided mode. Four typical cavity modes are shown individually in Fig.
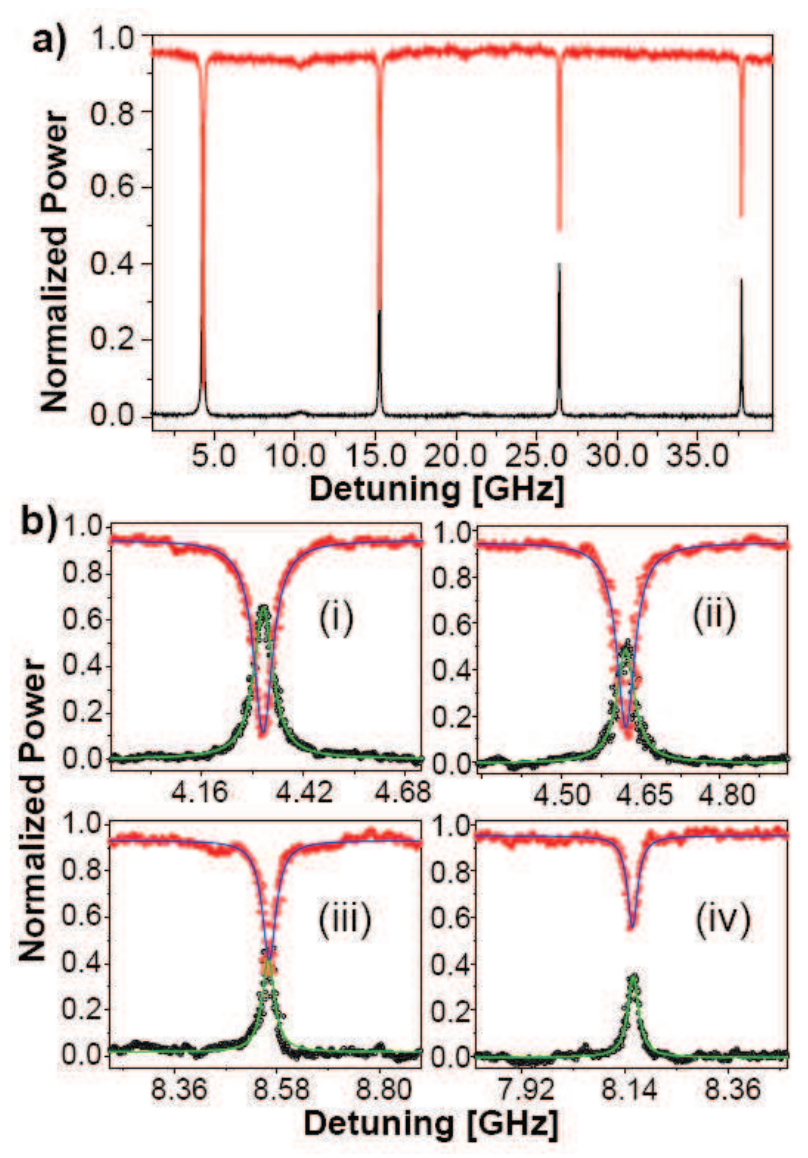

FIG. 3. (a) A typical part of the transmission (black curve) and reflection (red curve) spectra of the long nanofiber cavity for X-pol, showing periodically spaced cavity modes . (b) The transmission (black circles) and reflection (red circles) spectra for four typical cavity modes (i-iv) shown individually. The green and blue curves are the corresponding Lorentzian fits.

3(b). The data for the measured transmission and reflection spectra for the cavity modes are shown in black and red circles, respectively. The green and blue curves show the Lorentzian fits. The linewidths $(\kappa)$ for the modes marked as i, ii, iii and iv are 59, 41, 33 and $27 \mathrm{MHz}$ corresponding to the finesse $\left(F=\Delta \nu_{F S R} / \kappa\right)$ values of $175,252,314$ and 384 , respectively. One can notice that the on-resonance transmission $\left(T_{0}\right)$ and reflection $\left(R_{0}\right)$ is increasing and decreasing, respectively as the cavity linewidth is increased.

The amplitudes of the field transmission $(t)$ and reflection $(r)$ of a two-sided cavity can be formulated [20] as

$$
t=\frac{\sqrt{\kappa_{1} \kappa_{2}}}{\kappa / 2+i \Delta \varpi} ; \quad r=\frac{\frac{1}{2}\left(\kappa_{1}-\kappa_{2}-\kappa_{s}\right)-i \Delta \varpi}{\kappa / 2+i \Delta \varpi}
$$

where $\Delta \varpi$ is the detuning between the laser frequency and the cavity resonance, $\kappa_{s}$ is the intra-cavity loss rate, $\kappa_{1}$ and $\kappa_{2}$ are the coupling rates of the input and out- 

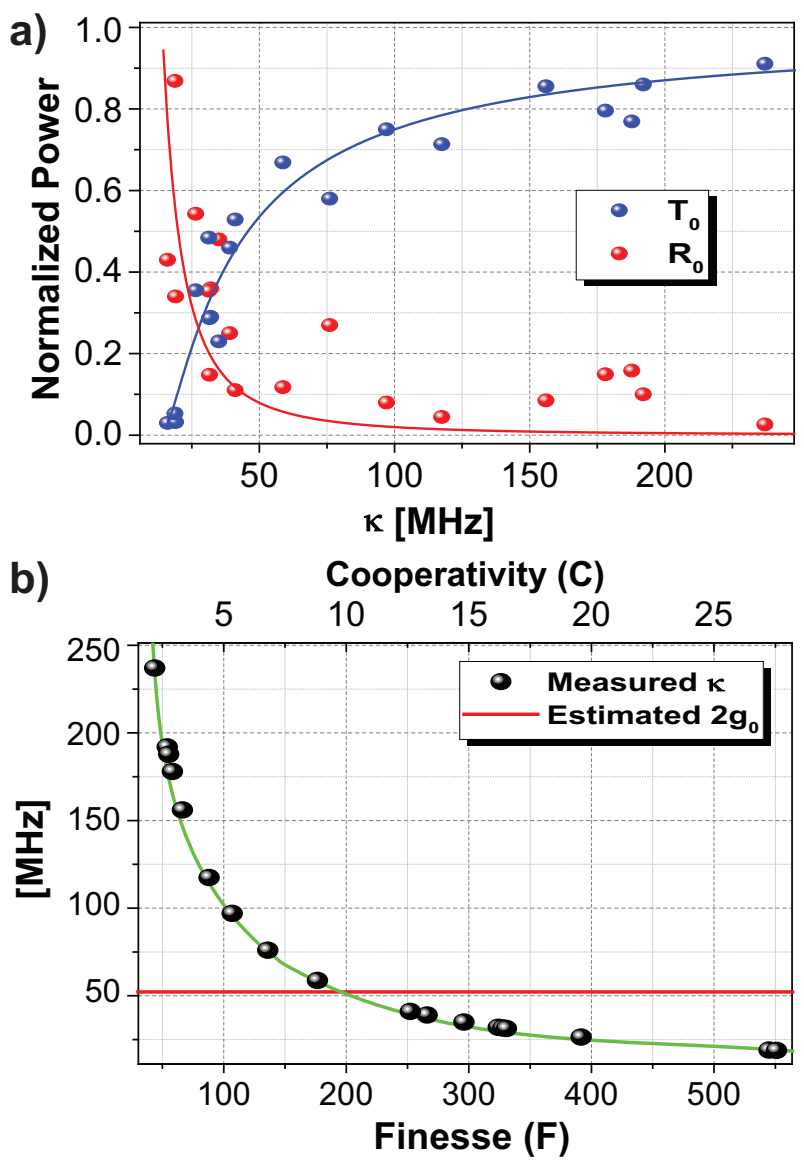

FIG. 4. (a) The measured on-resonance transmission, $\mathrm{T}_{0}$ (blue circles) and reflection, $\mathrm{R}_{0}$ (red circles) values vs the cavity linewidths $(\kappa)$ for typical cavity modes. The blue and red lines are the fits using Eq. 2. (b) The black circles show the measured cavity linewidths $(\kappa)$ vs the finesse $(F)$ values for the typical cavity modes. The green line is a fit showing the inverse relation. The red line shows the estimated single photon Rabi frequency $\left(2 g_{0}\right)$, assuming a Cs-atom trapped $200 \mathrm{~nm}$ away from the fiber surface. The corresponding single atom cooperativity $(C)$ is shown in the top axis.

put side mirrors, respectively and $\kappa=\kappa_{1}+\kappa_{2}+\kappa_{s}$ is the cavity linewidth. The power transmission $(T)$ and reflection $(R)$ from the cavity are given by $T=|t|^{2}$ and $R=|r|^{2}$. Assuming a symmetric cavity $\left(\kappa_{1}=\kappa_{2}=\kappa_{c}\right)$ the on-resonance $(\Delta \varpi=0)$ transmission and reflection can be written as

$$
T_{0}=\left|\frac{2 \kappa_{c}}{\kappa}\right|^{2}=\left|1-\frac{\kappa_{s}}{\kappa}\right|^{2} ; \quad R_{0}=\left|\frac{\kappa_{s}}{\kappa}\right|^{2}
$$

From the above equation it is clear that the $T_{0}$ and $R_{0}$ values will increase and decrease, respectively as the $\kappa$ increases. Moreover, when the $T_{0}$ and $R_{0}$ values are equal, the total out coupling rate $\left(2 \kappa_{c}\right)$ and the intra-cavity loss rate $\left(\kappa_{s}\right)$ balance each other and one can get $\kappa_{s}=\kappa / 2$.

Figure 4 (a) shows the $T_{0}$ (blue circles) and $R_{0}$ (red circles) values for the selected cavity modes (along with the modes shown in Fig. 3(b)) plotted against the corresponding $\kappa$ values. In the selection process we have chosen the cavity modes that have the highest transmission for the corresponding $\kappa$ values. The blue and red lines are the fits using Eq. 2. From the fits we estimate the lowest $\kappa_{s}$ value to be $15 \pm 1 \mathrm{MHz}$. Also one can clearly see from the plot that the $T_{0}$ and $R_{0}$ values are equal around $30 \mathrm{MHz}$. From this $\kappa_{s}$ value, we estimate the one-pass intra-cavity transmission to be $99.53 \%$. We have fabricated several cavity samples and measured the lowest $\kappa_{s}$ value to be in the range $15-20 \mathrm{MHz}$. This suggests that the fabrication process is reproducible. We must mention that we have also measured the cavity characteristics for the orthogonal polarization (Y-pol). We have found that the cavity transmission for $\mathrm{Y}$-pol is smaller compared to the $\mathrm{X}$-pol, resulting in higher $\kappa_{s}$ value.

Based on the optical characteristics of the cavity we now estimate the potential of the cavity in the context of cavity QED and quantum information application. Figure 4(b) summaries the measured $\kappa$ values corresponding to the $F$ values. The estimated $C$ and $2 g_{0}$ values are also shown assuming a single Cs-atom trapped $200 \mathrm{~nm}$ away from the fiber surface [8]. This shows that for the measured cavity modes we can achieve high cooperativity ranging from 3 to 25 . For cavity modes having $\kappa$ values smaller than $50 \mathrm{MHz}$ (i.e. finesse in the range 200 to 400), "strong-coupling" regime can be realized. As shown in Fig. 4(a), the on-resonance transmission for such cavity modes can be as high as $40 \%$ to $60 \%$. On the other hand, for the cavity modes having $\kappa$ values between $50 \mathrm{MHz}$ to $170 \mathrm{MHz}$, the on-resonance transmission range from $60 \%$ to $85 \%$, while maintaining a cooperativity of $3-10$. We must mention that using solid-state quantum emitters like quantum dot or color centers in nanodiamonds, the emitter can be placed directly on the nanofiber surface leading to much higher cooperativity in the range 1550 [8]. Hence such cavity modes can be implemented for fiber-based single photon sources and quantum nonlinear optics in "Purcell" regime.

In conclusion, we have demonstrated the fabrication of a $1.7 \mathrm{~cm}$ long nanofiber with highly uniform diameter of $500 \pm 2 \mathrm{~nm}$ over the entire length and maintaining high transmission of $>99 \%$. Furthermore, we fabricate two photonic crystal structures separated by $1.2 \mathrm{~cm}$ on such a nanofiber using femtosecond laser ablation, thus forming a centimeter-long nanofiber cavity. The high optical quality of such a cavity shows promising avenues for fiber-based quantum interface and single photon sources.

This work was supported by the Japan Science and Technology Agency (JST) as one of the Strategic Innovation projects. KPN acknowledges support from a grantin-aid for scientific research (Grant no. 15H05462) from the Japan Society for the Promotion of Science (JSPS). 
[1] H. J. Kimble, "The quantum internet," Nature 453, 1023-1030 (2008).

[2] P. Berman, Cavity Quantum Electrodynamics (Academic Press, Boston, 1994).

[3] A. Reiserer and G. Rempe, "Cavity-based quantum networks with single atoms and optical photons," Rev. Mod. Phys. 87, 1379-1418 (2015).

[4] J. McKeever, A. Boca, A. D. Boozer, J. R. Buck, and H. J. Kimble, "Experimental realization of a one-atom laser in the regime of strong coupling," Nature 425, 268-271 (2003).

[5] A. Boca, R. Miller, K. M. Birnbaum, A. D. Boozer, J. McKeever, and H. J. Kimble, "Observation of the vacuum Rabi spectrum for one trapped atom," Phys. Rev. Lett. 93, 233603 (2004).

[6] P. Maunz, T. Puppe, I. Schuster, N. Syassen, P. W. H. Pinkse, and G. Rempe, "Cavity cooling of a single atom," Nature 428, 50-52 (2004).

[7] T. G. Tiecke, J. D. Thompson, N. P. de Leon, L. R. Liu, V. Vuletić, and M. D. Lukin, "Nanophotonic quantum phase switch with a single atom," Nature 508, 241-244 (2014).

[8] F. L. Kien and K. Hakuta, "Cavity-enhanced channeling of emission from an atom into a nanofiber," Phys. Rev. A 80, 053826 (2009).

[9] J. D. Love, W. M. Henry, W. J. Stewart, R. J. Black, S. Lacroix, and F. Gonthier, "Tapered single-mode fibres and devices. Part 1: Adiabaticity criteria," IEE Proc. 138, 343-354 (1991)

[10] J. E. Hoffman, S. Ravets, J.A. Grover, P. Solano, P. R. Kordell, J. D. Wong-Campos, L. A. Orozco, S. L. Rolston, "Ultrahigh transmission optical nanofibers," AIP
Advances 4, 067124 (2014).

[11] K. P. Nayak, F. L. Kien, Y. Kawai, K. Hakuta, K. Nakajima, H. T. Miyazaki, and Y. Sugimoto, "Cavity formation on an optical nanofiber using focused ion beam milling technique," Opt. Express 19, 14040-14050 (2011).

[12] K. P. Nayak and K. Hakuta, "Photonic crystal formation on optical nanofibers using femtosecond laser ablation technique," Opt. Express 21, 2480-2490 (2013).

[13] K. P. Nayak, P. Zhang, and K. Hakuta, "Optical nanofiber-based photonic crystal cavity," Opt. Lett. 39, 232-235 (2014).

[14] M. Sadgrove, R. Yalla, K. P. Nayak, and K. Hakuta, "Photonic crystal nanofiber using an external grating," Opt. Lett. 38, 2542-2545 (2013).

[15] R. Yalla, M. Sadgrove, K. P. Nayak, and K. Hakuta, "Cavity quantum electrodynamics on a nanofiber using a composite photonic crystal cavity," Phys. Rev. Lett. 113, 143601 (2014).

[16] C. Wuttke, M. Becker, S. Brückner, M. Rothhardt, and A. Rauschenbeutel, "Nanofiber fabry-perot microresonator for nonlinear optics and cavity quantum electrodynamics," Opt. Lett. 37, 1949-1951 (2012).

[17] S. Kato and T. Aoki, "Strong coupling between a trapped single atom and an all-fiber cavity," Phys. Rev. Lett. 115, 093603 (2015).

[18] T. A. Birks and Y. W. Li, "Shape of fiber tapers," Journal of Lightwave Technology 10, 432 (1992).

[19] J. Keloth, M. Sadgrove, R. Yalla, and K. Hakuta, "Diameter measurement of optical nanofibers using a composite photonic crystal cavity," Opt. Lett. 40, 4122-4125 (2015).

[20] D. F. Walls and G. J. Milburn, Quantum Optics (Springer, Berlin, 2008). 